\title{
Two-level Topology Optimization for high definition material structures under the cgFEM framework
}

\author{
R. Merli*, E. Nadal*, J. Albelda*, J.J. Ródenas* \\ * Instituto de Investigación en Ingeniería Mecánica y Biomecánica (I2MB) \\ Universitat Politècnica de València \\ e-mail: \{ramegis, ennaso\}@upvnet.upv.es, \{jalbelda, jjrodena\}@mcm.upv.es
}

\begin{abstract}
Topology Optimization (TO) is a numerical tool for generating optimal structural topologies for holding specific load conditions. TO has gained popularity since the publication of SIMP by O. Sigmund [1] in 2001. SIMP algorithm proposes an optimal topology for a monolithic where the design variables (the material relative density at each element) are optimized. As you can appreciate, the number of variables is high and for real 3D problems it could get astronomic numbers, for instance in [2] authors consider $10^{9}$ design variables.

The underlying idea in this work is to split the monolithic domain in cells to perform the TO at each cell and not in the monolithic domain. Therefore, the optimization process could be performed in parallel, improving the performance of the method and not requiring of High Performance Computation centres. This idea has already being tackled by some authors [4], however the structural continuity between cells is an unsolved issue today. In this work we propose a method based of boundary stress equilibrium presented by P. Ladvèze [5] in order to apply the right boundary conditions to each cell for obtaining a geometrical continuity of the structure between cell. However, methodology presented in [5] is difficult to implement in the standard FE set. This issue has been addressed b using the Cartesian Grid Finite Element Method (cgFEM) [3]. cgFEM is equipped with a Cartesian mesh structure and a hierarchical data structure which strongly reduces the complexity of obtaining the equilibrated boundary conditions for each cell. The process presented in this work takes the following steps: $i$ ) a raw material distribution is carried out for the whole component. This could be done with few iterations of a TO process. ii) Using this information and the loads to which each cell is subjected to, a second TO process is launched at each cell defining the geometry of the same. Some results of the obtained topologies are presented in this work.
\end{abstract}

Acknowledgements The authors appreciate the financial support to this work of the Spanish Ministerio de Economía, Industria y Competitividad (DPI2017-89816-R).

\section{REFERENCES}

[1] O. Sigmund. A 99 line topology optimization code written in Matlab. Structural and Multidisciplinary Optimization. 21:120-127, 2001.

[2] N. Aage, E. Andreassen, B.S. Lazarov, O.Sigmund. Giga-voxel computational morphogenesis for structural design. Nature, 550(7674): 84-86, 2017.

[3] E. Nadal. Cartesian grid FEM (cgFEM): High performance h-adaptive FE analysis with efficient error control: application to structural shape optimization. Ph.D. Thesis, Universitat Politècnica de València, 2014.

[4] A. Ferrer, J. Oliver, J.C. Cante, O. Lloberas-Valls. Vademecum-based approach to multi-scale topological material design. Advanced Modeling and Simulation in Engineering Sciences. 3(1), 23, 2016.

[5] P. Ladevèze, E.A.W. Maunder. A general method for recovering tractions equilibrating element. Computer Methods in Applied Mechanics and Engineering, 137(2). 111-151, 1996. 